\title{
The role of the general practitioner and the out-of-hospital public rheumatologist in the diagnosis and follow-up of patients with polymyalgia rheumatica
}

\author{
A. Sobrero', C. Manzo², A. Stimamiglio ${ }^{3}$ \\ ${ }^{1}$ General Medicine, Liguria Region; \\ 2Service of Geriatric Rheumatology, Mariano Lauro-Sant'Agnello Hospital, ASL NA 3 Sud; \\ ${ }^{3}$ General Practitioner, ASL 3 Genovese, Italy
}

\section{SUMMARY}

Polymyalgia rheumatica (PMR) is a chronic inflammatory disease characterized by shoulder and pelvic girdle pain. Its onset peaks around the age of 75; the prevalence increases until the age of 90 and it is more frequent in females.

Diagnosis is mostly performed on the basis of symptoms. An increase of serum inflammatory markers is indicative, but not essential, while therapy is mainly based on glucocorticoids.

Since there is no universal agreement about diagnostic criteria for PMR, its detection is still difficult. There are discordant opinions about the fact that PMR can be recognised and managed by general practitioners (GPs), while patients with atypical features need to be referred to the rheumatologist.

In the Italian setting, the absence of recent epidemiological studies is associated with the total lack of a research protocol in primary care, from which relevant information could be derived. The out-of-hospital public rheumatologist is a peculiar figure of the Italian National Health System, who takes care of outpatients. Although differences between the different Italian regional health services exist, this professional figure has proved to be effective in reducing delay and increasing accuracy in PMR diagnosis.

Key words: Polymyalgia rheumatica; Primary care; Diagnosis.

Reumatismo, 2018; 70 (1): 44-50

\section{INTRODUCTION}

Dolymyalgia rheumatica (PMR) is a 1 common inflammatory disease affecting older adults. It is more prevalent in females and is mainly observed in the Caucasian ethnic group (1). It was first described by Bruce in 1888 (2). PMR classically occurs with sudden onset of bilateral myalgia/pain in the shoulder and hip girdles, as well as with systemic features, such as weight loss, nausea and fever, usually combined with raised inflammatory markers (1, $3,4)$. The pathogenesis of PMR is currently not well understood; infections and vaccinations (e.g., influenza and parainfluenza viruses) have occasionally been linked to a slightly higher risk of developing PMR (1). The general practitioner (GP) is usually the first physician who examines the PMR patient. He will undertake the differential diagnosis between PMR and other diseases that could mimic its presentation. In primary care, where the patient is already known for his/her previous comorbidities, a diagnostic and therapeutic workup is decided, according to his/her general clinical condition. Often, these decisions are taken not only on the basis of symptoms, but also of practical issues, such as the waiting time for a possible specialist's visit. In typical cases and when the referral cannot be done promptly, the GP will start treatment in an attempt to relieve the patient's pain. However, atypical presentations can occur in up to $20 \%$ of affected patients (5). Low-dose glucocorticoids (GC) are an effective treatment for most patients, often resulting in a striking improvement of symptoms, a feature which 
is considered an important diagnostic criterion of PMR by some authors (6).

\section{EPIDEMIOLOGY}

Data on the occurrence and manifestations of PMR should ideally rely on studies carried out in primary care, since most patients affected by PMR are diagnosed and treated in the setting of family medicine and are not referred to rheumatologists. A pivotal study from the UK (7) showed an age-adjusted incidence of PMR of 0.84/1000 persons per year. Another study (8) provided similar findings: in patients aged 50 years and older, the annual incidence of PMR was $1.13 / 1000$ persons, with a mean age at diagnosis of 75 years and women affected more frequently ( $75 \%$ of total cases). According to older studies, the annual PMR incidence would be about 0.12 cases $/ 1000$ persons $(9,10)$. A recent study based on cases of PMR identified by GPs (4) showed that prevalence of PMR increases until the age of 90, with a slight decrease thereafter. Very few Italian studies have addressed the epidemiology of PMR, in part because of the scarcity of established centralised database and electronic medical records. Manzo et al. (11) carried out a study on almost the whole population of a little town in the Italian Campania region, and found an annual incidence of 2.3 cases $/ 1000$ persons and a prevalence of $6.2 \% / 1000$ persons, again with a female predominance (70.5\% of patients). Salaffi et al. showed that musculoskeletal conditions are common in the general adult population of Italy and that the estimated prevalence of PMR was $0.37 \%$ (12).

The differences in incidence and prevalence in primary care studies could be related to several factors:

1. the different set of diagnostic criteria used for identifying patients;

2. the variability of expertise of different GPs regarding rheumatic diseases;

3. the diagnostic difficulty for patients with atypical presentations, such as those with normal erythrocyte sedimentation rate (ESR) (13).

Since GPs follow patients with PMR for their general health, they should consider that they have a higher cardiovascular risk than the general population, an observation shared with many other inflammatory rheumatic diseases. In a study performed in British practices (14), the risk of cardiovascular events was higher in PMR patients than in controls (36.1 vs 12.2 per 1000 person-years; adjusted hazard ratio 2.6, 95\% confidence interval 2.4-2.9). This study analysed 3249 PMR patients and 12,735 controls for 8 years.

In another study from UK (6), only $44.4 \%$ of patients with PMR were referred to specialist consultation. This information derives from a careful analysis of the centralized database of the practices in North Staffordshire. Disease codes and medical records, and in particular the results of laboratory examinations and the treatment prescribed, were used to corroborate the diagnosis. It is not clear if these results can be generalized to other areas, because North Staffordshire is a relatively deprived one. The percentage of specialist referral was as low as $17 \%$ in another paper (8). The incidence of PMR does not seem related to socioeconomic status (15).

\section{LABORATORY DIAGNOSIS}

PMR is mainly diagnosed according to clinical features because there are no specific laboratory tests $(16,17)$. However, even the clinical features are a combination of non-specific signs and symptoms, including aching and stiffness in the shoulder girdle, which fact can represent a complication in recognition of PMR.

In primary care, many studies suggest an improvement in the capacity of GPs in identifying common rheumatic diseases (18). GPs, as all clinicians, need continuous updating to gain more awareness in diagnosing rheumatic diseases, especially PMR. In fact, there is an inadequate use of investigation methods, which corresponds to a higher healthcare expense (18). Continuing education is a useful way to reduce the number of laboratory and radiological examinations requested before sending the patient to the specialist. 
According to Helliwell et al., it is possible to improve diagnosis with a more formalised collaboration between primary and secondary care. These guidelines reiterate that raised inflammatory markers are supportive but not essential to the diagnosis (6). A study from Mathew et al. found that about $91 \%$ of PMR patients have a raised ESR. The small proportion of patients with normal ESR usually has raised $\mathrm{C}$ reactive protein (CRP) values. Other abnormal blood results include normocytic anaemia and raised alkaline phosphatase (16). CRP is a more sensitive indicator of disease activity than ESR, as it is less affected by extraneous factors such as increasing age (19). Lastly, in the study by Terrazas et al., a significant number of patients presented with only mildly elevated ESR (20). Other abnormalities are normochromic, normocytic anaemia, increased alkaline phosphatase (over 1.5 times the normal level), and decreased albumin levels. Creatine kinase, rheumatoid factor and anti-cyclic citrullinated peptides antibodies are usually normal (20), which is useful for the differential diagnosis between PMR, rheumatoid arthritis and polymiositis. Checking baseline blood glucose and glycated haemoglobin may be useful if steroid treatment is likely to be started as a result of strongly suspected PMR (16).

\section{CLINICAL PRESENTATION IN GENERAL MEDICINE}

In a study by Helliwell et al. on 334 patients from primary care, $81.6 \%$ had symptoms and $45.7 \%$ showed shoulder girdle pain and muscular pain $(10.5 \%)$ (6). The fact than less than half of GP-diagnosed PMR patients showed shoulder pain, which is essential in classification criteria, emphasizes that they represent a different subset of PMR. Whether this is due to misclassification or to the presence of different presentations of the PMR syndrome is not clear.

Quick et al. found out that PMR is mostly diagnosed and managed in primary care, but observed that GPs have often trouble in identifying PMR (17). In this study, a review of 13 consecutive patients referred to hospital with PMR found that half of them had a different condition. When the records of 47 PMR patients from six practices were reviewed, $25 \%$ showed only a gradual symptom improvement after treatment, suggesting that perhaps PMR was not the correct diagnosis; $38 \%$ of them were eventually referred to hospital care for improved management (17). In other centres, identification of diseases other than PMR during follow up has been reported in up to a quarter of patients (17). In a study published by Helliwell et al. in 2013, in $38 \%$ of patients diagnosed in primary care, the GP did not adopt any of the known classification criteria; in many cases, no information has been provided as to how the PMR diagnosis had been supported (6).

Diagnostic accuracy is clearly essential, as misdiagnosis could result in prolonged inappropriate treatment with GC or, alternatively, a missed opportunity for early treatment of malignancy. Current diagnostic guidelines suggest sending the patient quickly to the specialist to confirm early diagnosis of patients with normal or very high inflammatory markers, atypical symptoms, poor response to corticosteroids or important systemic systems. In the study by Binard et al. (21), the main reasons for rheumatologist referrals were atypical PMR with GC dependency and difficulty in ruling out differential diagnoses, such as late-onset rheumatoid arthritis and calcium pyrophosphate dehydrate deposition disease. Diagnostic accuracy represents clearly an area where further primary carebased research is required $(6,16)$.

Binard et al. (21) found that there is an excellent agreement $(90.3 \%)$ between diagnosis of relapse made by GPs and by rheumatologists, as well as between diagnosis of relapse made by GPs and the subsequent decision to increase GC dosage $(95.1 \%$ agreement). Moreover, PMR activity score (PMR-AS), which is computed by summing five parameters: morning stiffness, ability to elevate the upper limbs, physician's global assessment using a 10-point visual analogue scale, pain measured by the patient using a 10-point visual analogue scale, and CRP, was highly sensitive and specific for diag- 
nosis of relapse made by rheumatologists, which represented the reference standard. The PMR-AS, determined by GPs, is a valid tool that would probably help GPs monitor and adjust GC regimens in patients with PMR. Both the shortage of rheumatologists and the predominant contribution of GPs to the management of PMR patients, lend considerable importance to these findings. Nevertheless, the percentage of patients managed by GPs or rheumatologists differs from one country to another and there is no clear explanation for these differences (21). Finally, Dejaco et al. suggested that clinical assessment of the hips and assessment of GC dose further improved specificity and sensitivity of defining remission and relapse in PMR, even if these features are not considered in PMR-AS (22).

\section{TREATMENT OF POLYMYALGIA RHEUMATICA}

The results on GPs' contribution to the management of patients with PMR in Brittany, France, are consistent with those obtained in Minnesota, USA (21): less than $40 \%$ of GPs routinely refer their patients to rheumatologists to confirm the diagnosis or monitor the treatment. Similarly, Mathew et al. found that more than $80 \%$ of patients are exclusively managed by their GP and there is a need to refer them to secondary care for further assessment if atypical features or a suboptimal response to treatment occur (16). Also, in the study by Aamer (23), it is suggested that PMR is mainly managed in primary care and less frequently in secondary care by rheumatologists and other specialists: in particular, referral to a rheumatologist should be considered in cases of age $<60$ years where chronic onset, lack of shoulder involvement or inflammatory stiffness, lack of response to GC or red flags, such as prominent systemic symptoms, weight loss or night pain are observed (23). In the study by Helliwell et al., moreover, PMR is identified as a disorder commonly managed in general practice, suggesting that in future research the inclusion of PMR patients recruited from primary care is necessary (6). If PMR ap- pears in its typical fashion, GPs may decide to start GC therapy without consulting the specialist. GPs habitually prescribe chronic GC treatment in other pathologies such as asthma or COPD. The use of NSAIDs or analgesic should be limited to the initial phases of the disease before the diagnosis is ascertained or in case of pain associated with other concomitant conditions.

When administering GC, physicians should consider possible contraindications, such as active mycobacterial infections, systemic mycotic infections, ocular Herpes or severe psychosis. Caution should be used when other comorbidities such as diabetes, hypertension, severe osteoporosis or glaucoma coexist in the patient with PMR. According to ACR/EULAR recommendations (24), treatment should start with a daily dosage of prednisone between 12.5 and $25 \mathrm{mg}$; a higher dosing regimen should be used in patients at high risk of relapse. Starting doses below $7.5 \mathrm{mg}$ of daily prednisone are discouraged. If response is not reached within two weeks, the daily dosage can be increased. After 4-8 weeks, prednisone should be decreased to $10 \mathrm{mg}$ and further tapered by 1-1.25 mg every month, until withdrawal. Prednisone is usually administered in a single daily dose in the morning. Sometimes a further evening administration can be used in case of severe night pain. A typical course of prednisone could last, in the best-case scenario, from 10 to 12 months. Table I shows an example of tapering, in a patient with a good clinical response, using the two formulations of immediate-release prednisone available in Italy. It is presently unknown if the treatment guidelines are followed in Italy both in clinical practice and among specialists. If GCs are used in patients with doubtful diagnosis, the correct diagnosis could be delayed and complicated.

In case of relapse, the dosing regimen prior to relapse should be re-established for 4-8 weeks.

Factors linked with relapses have still to be determined. According to some studies, relapses are associated with the female sex (25), ESR > $40 \mathrm{~mm} / \mathrm{h}$ (26) and peripheral arthritis (27). An increase of the inflamma- 
Table I - Suggested tapering of prednisone.

\begin{tabular}{|c|l|}
\hline Month & Daily dose of prednisone \\
\hline 1 & $12.5 \mathrm{mg}$ (half a $25 \mathrm{mg}$-tablet) \\
\hline 2 & 11.25 (two $5 \mathrm{mg}$-tablets and two and half $5 \mathrm{mg}$ tablets on alternate days) \\
\hline 3 & $10 \mathrm{mg}$ (two $5 \mathrm{mg}$-tablets) \\
\hline 4 & $\begin{array}{l}8.75 \mathrm{mg} \text { (two } 5 \mathrm{mg} \text {-tablets and one and half } 5 \mathrm{mg} \text { - tablets on alternate } \\
\text { days) }\end{array}$ \\
\hline 5 & $7.5 \mathrm{mg}$ (one and half $5 \mathrm{mg}$-tablets) \\
\hline 6 & $6.25 \mathrm{mg}$ (a quarter of a $25 \mathrm{mg}$-tablet) \\
\hline 7 & $5 \mathrm{mg}$ (one $5 \mathrm{mg}$-tablet) \\
\hline 8 & 3.75 (one $5 \mathrm{mg}$ - tablet and half a 5 mg-tablet on alternate days) \\
\hline 9 & $2.5 \mathrm{mg}$ (half a $5 \mathrm{mg}$-tablet) \\
\hline 10 & $1.25 \mathrm{mg}$ (half a $5 \mathrm{mg}$-tablet on alternate days) \\
\hline
\end{tabular}

tory indexes, without clinical symptoms of PMR, is not sufficient to define a relapse, but GPs often do not follow this advice. PMR patients, in consideration of their mean age, have many other causes of articular pain; conversely relapses of PMR can occur with clinical features differing from the initial presentation, such as RS3PE or peripheral arthritis.

Initiation of treatment by GPs before blood examinations is another error which could complicate the follow-up, especially if therapy is not effective and the patient is referred to a specialist. A too rapid tapering is another common attitude that favours relapses. Italian GPs sometimes use deflazacort, the efficacy of which seems to be similar to that of prednisone (28-30). However, the benefit of deflazacort in terms of side effects is unclear, it is not reimbursed by the Italian National Health System, and is more expensive than prednisone.

GPs are not familiar with the use of methotrexate as steroid sparing agent $(31,32)$. In addition, its use in PMR is off-label.

THE ROLE OF THE OUT-OF-HOSPITAL SETTING

Although considered the most frequent inflammatory rheumatic disease in the el-

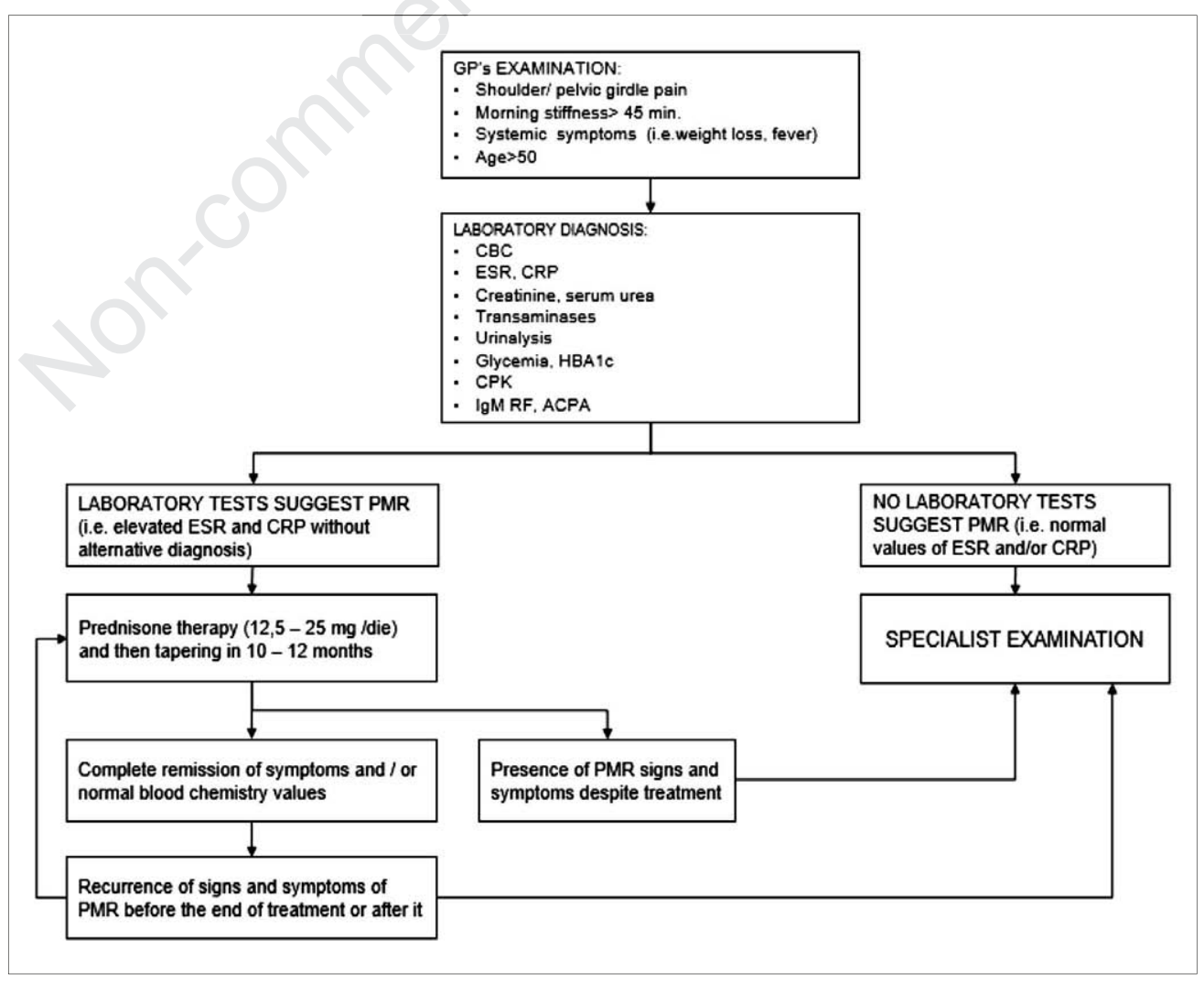

Figure 1 - Flow-chart of the shared treatment of PMR patients between GPs and rheumatologists. 
derly, PMR is scarcely present in the experience of the GPs $(33,34)$. It can be estimated that a GP with 1500 patients can experience on average 1-2 cases of PMR every year (11). This may justify the low level of familiarity that the GP has with PMR. Moreover, the absence of elements that can allow a diagnosis of certainty contributes to making PMR a sort of magic cauldron in which all forms of arthromyalgia associated with elevation of inflammatory indexes that rapidly respond to low dosages of prednisone are included (35). It is also possible that a minority of PMR patients go into remission even in the absence of properly administered corticosteroid therapy (2).

In Italy, there is a specific professional figure represented by the out-of-hospital public rheumatologist, who is not present in other countries. He may represent a link between the GP and the centres of second or third level. The capability of this specialist to intercept a large proportion of patients with PMR is directly proportional to the level of collaboration that has been established with the GPs. When this collaboration is present, more patients with PMR are also visited by the rheumatologist (36-38).

In addition, the out-of-hospital public specialist is the only specialist who can also perform a domiciliary rheumatological visit if this is requested by the GP. This is particularly helpful in the case of very old patients and disabled ones. However, the level and modalities of this organization are not homogeneous all over the country (Figure 1).

\section{CONCLUSIONS}

The assessment of the role of the territory in the diagnosis and follow up of the patient with PMR has highlighted some critical issues. A shared pathway between GPs, out-of-hospital public rheumatologists, hospital and university rheumatologists may improve timing and precision of diagnosis. These points should be included in the working agenda of the various scientific societies involved.

\section{REFERENCES}

1. Tshimologo M, Saunders B, Muller S, et al. Patients' views on the causes of their polymyalgia rheumatica: a content analysis of data from the PMR Cohort Study. BMJ Open. 2017; 7: e014301.

2. Bruce W. Senile rheumatic gout. BMJ 1888; 2: 811-3.

3. Muller S, Hider SL, Helliwell T, et al. Characterising those with incident polymyalgia rheumatica in primary care: results from the PMR Cohort Study. Arthritis Res Ther. 2016; 18: 200.

4. Yates M, Graham K, Watts RA, MacGregor AJ. The prevalence of giant cell arteritis and polymyalgia rheumatica in a UK primary care population. BMC Musculoskelet Disord. 2016; 17: 285.

5. Twohig H, Mitchell C, Mallen C, et al. "I suddenly felt I'd aged": a qualitative study of patient experiences of polymyalgia rheumatica (PMR). Patient Educ Couns. 2015; 98: 645-50.

6. Helliwell T, Hider SL, Mallen CD. Polymyalgia rheumatica: diagnosis, prescribing, and monitoring in general practice. Br J Gen Pract. 2013; 63: e361-6.

7. Smeeth L, Cook C, Hall AJ. Incidence of diagnosed polymyalgia rheumatica and temporal arteritis in the United Kingdom, 1990-2001. Ann Rheum Dis. 2006; 65: 1093-8.

8. Barraclough K, Liddell WG, du Toit J, et al. Polymyalgia rheumatica in primary care: a cohort study of the diagnostic criteria and outcome. Fam Pract. 2008; 25: 328-33.

9. Cimmino MA, Zaccaria A. Epidemiology of polymyalgia rheumatica. Clin Exp Rheumatol. 2000; 18: S9-11.

10. Salvarani C, Macchioni PL, Tartoni PL, et al. Polymyalgia rheumatica and giant cell arteritis: a 5-year epidemiologic and clinical study in ReggioEmilia, Italy. Clin Exp Rheumatol. 1987; 5: 205-15.

11. Manzo C, Balduccelli M, Cappiello F, et al. Prevalence and incidence of polymyalgia rheumatica in Massa Lubrense, Italy. Trends Med 2009; 9: 85-9.

12. Salaffi F, De Angelis R, Grassi W, MArche Pain and study, INvestigation Group (MAPPING). Prevalence of musculoskeletal conditions in an Italian population sample: results of a regional community-based study. I. The MAPPING study. Clin Exp Rheumatol. 2005; 23: 819-28.

13. Ellis ME, Ralston S. The ESR in the diagnosis and management of the polymyalgia rheumatica/giant cell arteritis syndrome. Ann Rheum Dis 1983; 42: 168-70.

14. Hancock AT, Mallen CD, Muller S, et al. Risk of vascular events in patients with 
polymyalgia rheumatica. CMAJ. 2014; 186: E495-501.

15. Hayward RA, Rathod T, Muller S, et al. Association of polymyalgia rheumatica with socioeconomic status in primary care: a crosssectional observational study. Arthritis Care Res (Hoboken). 2014; 66: 956-60.

16. Mathew R, Rashid A. Polymyalgia rheumatica in primary care: managing diagnostic uncertainty. BMJ. 2015 7; 351: h5199.

17. Quick V, Kirwan JR. Our approach to the diagnosis and treatment of polymyalgia rheumatica and giant cell (temporal) arteritis. J R Coll Physicians Edinb. 2012; 42: 341-9.

18. S. Bahlas, C. Ramos-Remus, P. Davis. Utilisation and costs of investigations, and accuracy of diagnosis of polymyalgia rheumatica by family physicians. Clin Rheumatol. 2000; 19: 278-80.

19. Van Hecke O. Polymyalgia rheumatica - diagnosis and management. Aust Fam Physician. 2011; 40: 303-6.

20. Terrazas D, Schumann L. Managing polymyalgia rheumatica and giant cell arteritis in the primary care setting. J Am Acad Nurse Pract. 1997; 9: 289-92.

21. Binard A, Lefebvre B, De Bandt M, et al. Validity of the polymyalgia rheumatica activity score in primary care practice. Ann Rheum Dis. 2009; 68: 541-5.

22. Dejaco C, Duftner C, Cimmino MA, et al. Polymyalgia rheumatica: clinical update. Definition of remission and relapse in polymyalgia rheumatica: data from a literature search compared with a Delphi-based expert consensus. Ann Rheum Dis. 2011; 70: 447-53.

23. Ameer F, McNeil J. Polymyalgia rheumatica: clinical update. Aust Fam Physician. 2014; 43: 373-6.

24. Dejaco C, Singh YP, Perel P, et al. 2012 Recommendations for the management of polymyalgia rheumatica: a European League Against Rheumatism/American College of Rheumatology collaborative initiative. Ann Rheum Dis. 2015; 74: 1799-80.

25. Cimmino MA, Parodi M, Caporali R, Montecucco $\mathrm{C}$. Is the course of steroid-treated polymyalgia rheumatica more severe in women? Ann N Y Acad Sci. 2006; 1069: 315-21.

26. Cantini F, Salvarani C, Olivieri I, et al. Erythrocyte sedimentation rate and C-reactive protein in the evaluation of disease activity and severity in polymyalgia rheumatica: a prospective follow-up study. Semin Arthritis Rheum. 2000; 30: 17-24.

27. Salvarani C, Cantini F, Macchioni P, et al.
Distal musculoskeletal manifestations in polymyalgia rheumatica: a prospective followup study. Arthritis Rheum. 1998; 41: 1221-6.

28. Krogsgaard MR, Lund B, Johnsson B. A longterm prospective study of the equipotency between deflazacort and prednisolone in the treatment of patients with polymyalgia rheumatica. J Rheumatol. 1995; 22: 1660-2.

29. Di Munno O, Imbimbo B, Mazzantini M, et al. Deflazacort versus methylprednisolone in polymyalgia rheumatica: clinical equivalence and relative antiinflammatory potency of different treatment regimens. J Rheumatol. 1995; 22: 1492-8.

30. Cimmino MA, Moggiana G, Montecucco C, et al. Long term treatment of polymyalgia rheumatica with deflazacort. Ann Rheum Dis. 1994; 53: 331-3.

31. Caporali R, Cimmino MA, Ferraccioli G, et al. Systemic Vasculitis Study Group of the Italian Society for Rheumatology. Prednisone plus methotrexate for polymyalgia rheumatica: a randomized, double-blind, placebocontrolled trial. Ann Intern Med. 2004; 141: 493-500.

32. Ferraccioli G, Salaffi F, De Vita S, et al. Methotrexate in polymyalgia rheumatica: preliminary results of an open, randomized study. J Rheumatol. 1996; 23: 624-8.

33. Hutchings A, Hollywood J, Lamping DL, et al. Clinical outcomes, quality of life and diagnostic uncertainly in the first year of polymyalgia rheumatica. Arthritis Care Res. 2007; 57 : 803-9.

34. Maradit Kremers H, Reinalda MS, Crowson $\mathrm{CS}$, et al. Use of physician services in a population based cohort of patients with polymyalgia rheumatica over the course of their disease. Arthritis Care Res. 2005; 53: 395-403.

35. Camellino D, Cimmino MA. Are the new ACR/EULAR criteria the ultimate answer for polymyalgia rheumatica classification? J Rheumatol 2016; 43: 836-8.

36. Manzo C, Natale M. Polymyalgia rheumatica and cancer risk: the importance of the diagnostic set. Open Access Rheumatol. 2016; 8: 93-5.

37. Manzo C. Widespread headache as the first clinical manifestation of giant cell arteritis in patients affected by polymyalgia rheumatica. Reumatologia. 2016; 54: 236-8.

38. Manzo C, Natale M, Cappiello F. Quanti anziani con polimialgia reumatica passano sotto gli occhi del medico del territorio? Ger Extraosp. 2008; VI: 12-5. 